\title{
OBTENÇÃO DE DIDÍMIO METÁLICO A PARTIR DOS ÓXIDOS DE TERRAS RARAS PRODUZIDOS EM ARAXÁ, BRASIL*
}

\author{
João Batista Ferreira Netor ${ }^{1}$ \\ Fernando José Gomes Landgrar ${ }^{2}$ \\ Gerhard Ett ${ }^{3}$ \\ Celia Aparecida Lino dos Santos ${ }^{4}$ \\ Andre Luiz Nunis da Silva ${ }^{5}$ \\ João Ricardo Filipini da Silveira ${ }^{6}$ \\ Fabiana Yamasaki Martins Vieira ${ }^{7}$ \\ Maciel dos Santos Luz
}

\section{Resumo}

Didímio metálico foi obtido no Instituto de Pesquisas Tecnológicas do Estado de São Paulo, a partir do óxido de didímio, o qual foi produzido pela extração por solventes de óxidos de terras raras do concentrado obtido na mina de nióbio da Companhia Brasileira de Metalurgia e Mineração - CBMM. Foram investigadas duas rotas tecnológicas, metalotérmica e redução eletroquímica, ambas ocorrendo em sais fundidos. Foi possível produzir didímio com um mínimo de $99 \%$ de pureza por ambas as rotas, mas a rota metalotérmica demanda uma etapa de processamento adicional para remover impurezas e agentes redutores.

Palavras-chave: Terras Raras; Didimio; Eletrolise; Metalotermia.

\section{DIDYMIUM OBTAINMENT FROM RARE EARTH OXIDES PRODUCED IN ARAXÁ, BRAZIL}

\section{Abstract}

Metallic Didymium was obtained at the São Paulo Institute for Technological Research (IPT), from didymium oxide, which was produced by solvent extraction of rare earth oxides processed from a rare earth concentrate produced by Companhia Brasileira de Metalurgia e Mineração - CBMM - from its niobium mine. Two routes were investigated, metallothermic and electrochemical reduction, both in molten salts. Both routes allowed didymium obtainment with $99 \%$ minimum purity, but the metallothermic route needs an additional processing step to remove the excess of reducing agent..

Keywords: Rare Earth; Didymium; Electrolysis; Metallothermia.

1 Engenheiro Metalurgista, Doutor, Pesquisador/Diretor de Centro, LPM/CTMM, Instituto de Pesquisas Tecnológicas do Estado de São Paulo, Brasil.

2 Engenheiro Metalurgista, Doutor, Diretor Presidente, Instituto de Pesquisas Tecnológicas do Estado de São Paulo, Brasil.

3 Engenheiro Químico, Doutor, Pesquisador, Instituto de Pesquisas Tecnológicas do Estado de São Paulo, Brasil.

4 Química, Doutor, Pesquisadora, LCP/CTMM, Instituto de Pesquisas Tecnológicas do Estado de São Paulo, Brasil.

5 Engenheiro Químico, Mestre, Pesquisador, LPM/CTMM, Instituto de Pesquisas Tecnológicas do Estado de São Paulo, Brasil..

6 Engenheiro de Materiais, Mestre, Pesquisador, LPM/CTMM, Instituto de Pesquisas Tecnológicas do Estado de São Paulo, Brasil.

7 Química, Pesquisadora, LCP/CTMM, Instituto de Pesquisas Tecnológicas do Estado de São Paulo, Brasil.

8 Químico, Doutor, Pesquisadora, LPM/CTMM, Instituto de Pesquisas Tecnológicas do Estado de São Paulo, Brasil.. 


\section{INTRODUÇÃO}

Neodímio ou didímio (neodímio + praseodímio) são considerados metais de terras raras vitais para a produção de imãs utilizados em discos rígidos, carros elétricos e híbrido, celulares e turbinas eólicas [1]. Atualmente, a China é responsável pela produção de quase $90 \%$ dos elementos de terras raras ETR. Recentemente, os chineses restringiram as exportações de ETR, o que representou riscos ao fornecimento ao resto do mundo. O país asiático é especialista em extração por solventes, separação dos óxidos dos diferentes metais, na redução em metais de terras raras e na produção de imãs de terras raras [2]. Baseado nesse cenário, muitas empresas de diferentes países estão procurando por alternativas de fornecimento fora da China. Depois da China, o Brasil possui a segunda maior reserva conhecida: 22 milhões de toneladas [3], como mostra a Tabela 1.

Tabela 1. Reservas mundiais de Terras Raras [3].

\begin{tabular}{|l|l|}
\hline País & $\begin{array}{l}\text { Reservas } \\
(\mathbf{t})\end{array}$ \\
\hline Estados Unidos & 1.800 .000 \\
\hline Austrália & 3.200 .000 \\
\hline Brasil & 22.000 .000 \\
\hline China & 55.000 .000 \\
\hline Índia & 3.100 .000 \\
\hline Malásia & 30.000 \\
\hline Outros Países & 41.000 .000 \\
\hline $\begin{array}{l}\text { Total mundial } \\
\text { (aproximadament } \\
\text { e) }\end{array}$ & 130.000 .00 \\
\hline
\end{tabular}

A Figura 1 mostra os depósitos mais importantes do país [4].

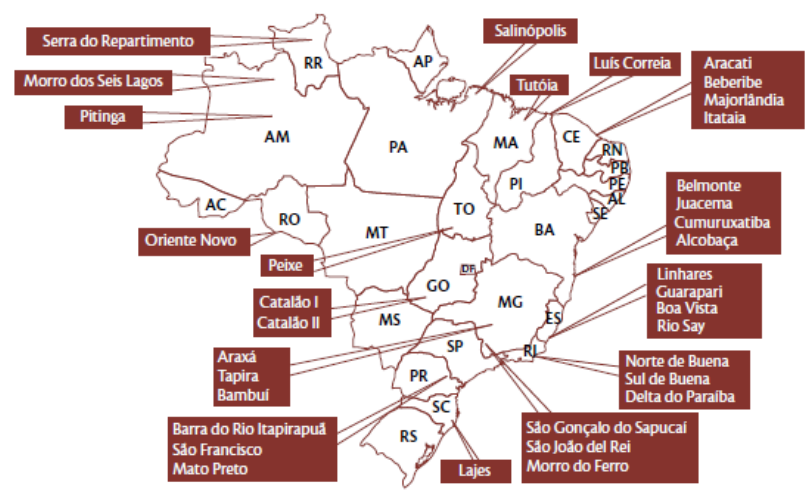

Figura 1 Principais depósitos de minérios de Terras Raras no Brasil [4].

Destaca-se o depósito de Terras Raras pesadas (Xenótimo) da companhia Mineração Taboca, localizada em Pitinga no estado do Amazonas, pertencente ao grupo peruano Minsur. Outro destaque é a empresa Mineração Serra Verde do grupo Mining Ventures Brazil, que pretende explorar num futuro próximo o depósito localizado na cidade de Minaçu, GO.

Contudo, a iniciativa mais importante nesse momento no país é indiscutivelmente $p$ projeto da Companhia Brasileira de Metalurgia e Mineração - CBMM, a qual explora 
nióbio em sua mina localizada próximo a cidade mineira de Araxá. O mineral monazita, que é um fosfato de ETR, é encontrado no rejeito da extração do minério de nióbio.

A concentração de monazita no minério da CBMM é quase a mesma do mineral em qual o nióbio é encontrado (Bariopirocloro).

Tab 2. Composição mineralológica media do minério residual de Araxá [5].

\begin{tabular}{|l|l|}
\hline Mineral & $\%$ \\
\hline Bariopirocloro & 4 \\
\hline Limonite, goetite & 36 \\
\hline Barita & 20 \\
\hline Magnetita & 16 \\
\hline Gorceixita & 6 \\
\hline Monazita & 4 \\
\hline Ilmenita & 5 \\
\hline Quartzo & 4 \\
\hline Outros & 5 \\
\hline Total & 100 \\
\hline
\end{tabular}

A vantagem na exploração de monazita é que o mineral é encontrado no rejeito do minério de nióbio, eliminando a necessidade de mineração adicional. A CBMM investiu aproximadamente $R \$ 60$ milhões para desenvolver a etapa de concentração do mineral de terras raras (para uma capacidade de 3000 t/ano) e atualmente se concentram no processo de separação de óxidos de terras raras. A separação é feita pelo processo de extração por solventes, cuja atual planta piloto é capaz de processar 5 t/ano de quatro produtos: óxido de cério, óxido de lantânio, óxido de terras raras pesadas e óxido de didímio, esse último uma mistura de óxido de praseodímio e neodímio.

O IPT (Instituto de Pesquisas Tecnológicas do Estado de São Paulo) tem estudado a redução do óxido de didímio produzido pela CBMM por duas rotas: redução metalotérmica e por eletrólise, ambas realizadas em sais fundidos. O objetivo desse artigo é apresentar alguns resultados preliminares desse estudo.

\section{MATERIAIS E MÉTODOS}

\subsection{Redução metalotérmica}

Neodímio e praseodímio são muito estáveis e podem ser reduzidos por poucos elementos. Para esse caso, cálcio metálico foi selecionado como agente redutor devido ao óxido de cálcio ser mais estável que o óxido de praseodímio e neodímio. A redução direta de óxido de TR não é recomendada pois a reação é altamente exotérmica, o que poderia resultar em sinterização dos produtos e reagentes, isto é, do óxido de TR, $\mathrm{CaO}$ e elementos de terras raras fundidos. A camada de $\mathrm{CaO}$, formada como produto da reação, pode reagir com o óxidos de terras raras formando compostos estáveis que podem se tornar uma barreira para a difusão e levar a uma reação incompleta. Assim, a redução calciotérmica foi realizada em um banho de sal fundido constantemente agitado, para garantir a dissipação térmica e a dissolução do óxido de cálcio, prevenindo o acumulo na interface entre o óxido de TR e o cálcio. Além do mais, cálcio é solúvel no sal fundido e assim sua atividade pode ser controlada, evitando o contato direto entre o óxido de terra rara e o cálcio 
metálico. Para a realização desses testes, foi escolhido cloreto de cálcio como sal fundido, pois ele possui alta solubilidade para $\mathrm{Ca}$ e $\mathrm{CaO}[6,7]$.

Primeiramente, aproximadamente $5 \mathrm{~kg}$ de $\mathrm{CaCl} 2$ misturado com cálcio metálico, em excesso considerando a reação estequiométrica, foram adicionados a um reator fechado, onde a mistura foi desidratada sob vácuo a $350{ }^{\circ} \mathrm{C}$ por 12 horas e a $650{ }^{\circ} \mathrm{C}$ por 3 horas.

O reator foi então preenchido com argônio para assim evitar a oxidação do cálcio metálico. A pressão foi mantida levemente superior a pressão atmosférica (aproximadamente 1000 mbar absoluto) para prevenir a entrada de ar no reator e uma evaporação excessiva do sal fundido.. O sal foi fundido e o reator aquecido à $1050{ }^{\circ} \mathrm{C}$. Essa temperatura está acima da temperatura de fusão do $\mathrm{Nd}$ e $\operatorname{Pr}\left(1024^{\circ} \mathrm{C}\right.$ e $935^{\circ} \mathrm{C}$, respectivamente) e foi definida para facilitar a coleta didímio metálico, já que as gotas poderiam para um pequeno cadinho coletor, conforme é representado pela Figura 2.

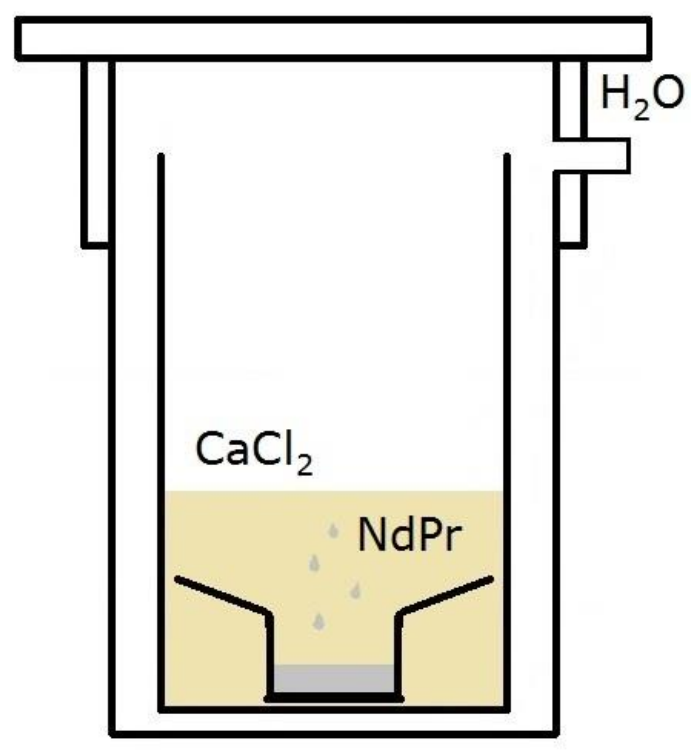

Figura 2. Esquema experimental para redução metalotérmica.

A tampa do reator possui um sistema de arrefecimento por água e contém um silo com óxido de didímio que poderá ser alimentado ao sal fundido por uma rosca sem fim. Após a fusão do sal, aproximadamente $500 \mathrm{~g}$ de óxido de didímio é alimentado ao reator a uma taxa controlada, enquanto o banho é agitado. Ao final do ensaio, o agitador é desligado e, após um determinado tempo para deposição metal, o reator resfriado.

O reator foi então aberto, o sal removido e, finalmente, o cadinho coletor com o didímio foi retirado. O metal recuperado foi pesado e analisado por SEM-EDS e ICPMS (7900, Agilent), Carbono foi determinado por combustão direta e detecção por infravermelho (CS-300, Leco) e oxigênio foi determinado por fusão em atmosfera inerte (ONH836, Leco).

O metal foi então refundido e refinado sob vácuo para a remoção do excesso de cálcio dissolvido no didímio. Após o refino, foram realizadas novamente as mesmas análises.

\subsection{Voltametria cíclica}


Os experimentos de voltametria cíclica foram realizados com um potenciostato do tipo PAR-Princeton Aplied Research, modelo PARstat 4, controlado pelo software VersaStudio 2.42. O ensaio foi realizado num reator de inconel e um cadinho de grafite de alta densidade para os sais fundidos. Foram utilizados três eletrodos: hastes de Mo (6 mm e $3 \mathrm{~mm}$ de diâmetro) como eletrodo de trabalho, haste de grafite de alta densidade (12 $\mathrm{mm}$ de diâmetro) como eletrodo auxiliar e foi de $\mathrm{Pt}$ $(0,15 \mathrm{~mm}$ de diâmetro) como eletrodo de quase-referencia.

Foram estudadas as seguintes misturas de sais fundidos (eletrólitos): LiF-CaF $2^{-}$ $\mathrm{NdF}_{3}-\mathrm{Nd}_{2} \mathrm{O}_{3}$, LiF-CaF $2-\mathrm{PrF}_{3}-\mathrm{Pr}_{11} \mathrm{O}_{6}$ e $\mathrm{LiF}-\mathrm{CaF}_{2}-\mathrm{NdF}_{3}-\mathrm{PrF}_{3}-\mathrm{Nd}_{2} \mathrm{O}_{3}-\mathrm{Pr}_{6} \mathrm{O}_{11}$. A última mistura foi utilizada para simular o óxido de didímio. A mistura de sais foi desidratada por 12 horas a $350^{\circ} \mathrm{C}$ e por 3 horas a $650{ }^{\circ} \mathrm{C}$, sob vácuo. $\mathrm{O}$ reator foi então preenchido com argônio e a mistura de sais aquecida até $820^{\circ} \mathrm{C}$.

Os experimentos exploraram a faixa de estabilidade de potencial do eletrólito para diferentes taxas de varreduras.

\subsection{Redução Eletroquímica}

Os ensaios ocorreram sob uma atmosfera controlada de argônio num reator de inconel. Foram utilizadas por ensaio aproximadamente $14 \mathrm{~kg}$ de uma mistura de fluoretos ( $\left(\mathrm{LiF}-\mathrm{CaF}_{2}-\mathrm{NdF}_{3}-\mathrm{PrF}_{3}\right)$ em proporção definida. Antes de cada ensaio os sais eram desidratados por processo semelhante ao descrito nos ensaios anteriores. Após desidratação, os sais eram fundidos e aquecidos a temperaturas variando de 1020 a $1050{ }^{\circ} \mathrm{C}$ em um cadinho de grafite de alta densidade. O catodo de W ou Mo foi imerso no banho de sais fundidos e rodeado por um anodo cilíndrico feito de grafite de alta densidade. Entre o catodo e o anodo, foi instalado um eletrodo de $\mathrm{Pt}$ (quase-referencia). Para coleta do metal produzido, um pequeno cadinho de Mo ou $\mathrm{Nb}$ foi instalado abaixo do catodo e imerso ao sal fundido. Esse cadinho foi utilizado para coleta do didímio metálico produzido. O sistema foi aquecido por um forno tipo poço já que a corrente aplicada não era suficiente para manter o sistema aquecido a temperatura de trabalho.

A tampa do reator possui um silo em que mais ou menos $420 \mathrm{~g}$ de óxido de didímio foram adicionados ao sal fundido a uma determinada taxa através de um sistema de rosca sem fim. A célula operou no modo galvanostático, com correntes variando entre 80 e $150 \mathrm{~A}$, dependendo da concentração e da taxa de alimentação de óxido de didímio. A tensão da célula variou entre $3,5 \mathrm{~V}$ a $5 \mathrm{~V}$, dependendo da concentração de óxido no sal fundido. A densidade de corrente variou entre 0,3 a 0,5 $\mathrm{A} / \mathrm{cm}^{2}$ no anodo e 4 a $6 \mathrm{~A} / \mathrm{cm}^{2}$ no catodo.

Como $\mathrm{CO}$ e $\mathrm{CO}_{2}$ eram gerados no anodo durante a eletrólise, assim como algum perfluorcarbono, foi instalado um sistema de análise e tratamento dos gases a jusante do reator. Eventuais perfluorcarbonos eram absorvidos por um selo hidráulico e monitorados por alterações no pH. Já o $\mathrm{CO}$ e $\circ \mathrm{CO}_{2}$ foram analisados por analisador infravermelho não-disperivo NDIR (Madur GA-60).

\section{RESULTADOS E DISCUSSÃO}

\subsection{Redução Calciotérmica}

Para a redução calciotérmica, a conversão média foi de $90 \%$. Supõe-se que os 10 $\%$ restantes do metal reduzido ficou no banho de sal fundido distribuído como uma "névoa" de gotículas que não coalesceram, permanecendo disperso pelo $\mathrm{CaCl}_{2}$. Contudo, a quantidade de metal no cadinho coletor foi menor do que o esperado, 
correspondendo a apenas $50 \%$ do óxido total alimentado. Isto pode ser explicado pelo fato de parte das gotículas de metal terem fluido para fora do funil anexo ao cadinho coletor, depositando assim no fundo do reator.

A solubilidade do $\mathrm{CaO}$ é limitada a $10 \%$ no cloreto de cálcio. Logo, há um limite para quanto óxidos de TR podem ser reduzidos, sem que haja precipitação de $\mathrm{CaO}$, o qual poderia contaminar o sal fundido reduzido. Isso faz com que o processo tenha que ocorrer em bateladas.

Na Figura 3, podem ser identificados dois tipos de precipitados na microestrutura das amostras de didímio, representados pelos pontos 1 e 5 (áreas escuras) e pelo ponto 2. Como pode ser visto na Tabela 3, os pontos 1 e 5 são ricos em $\mathrm{Ca}$, provavelmente precipitado, enquanto o ponto 2 é rico em Al, provavelmente pela formação do intermetálico $(\mathrm{Nd}, \mathrm{Pr}) \mathrm{Al}_{3}$. As áreas 3 e 4 são representativas ao metal produzido, aos quais são encontrados $\mathrm{Nd}$ e $\mathrm{Pr}$ em proporções muito próximas aos encontradas no óxido de didímio, com alguma contaminação do $\mathrm{Ca}$ e algumas impurezas encontradas no óxido de didímio, no cálcio metálico e no cloreto de sódio, tal como Al.

A análise de oxigênio não foi considerada na análise EDS, pois é significativamente difícil inserir a amostra no microscópio sem alguma oxidação da superfície. Assim, considera-se que a concentração de oxigênio venha apenas da preparação da amostra. Isso pôde ser comprovado pelo fato de apenas 20 ppm de oxigênio terem sido determinados por análise química.

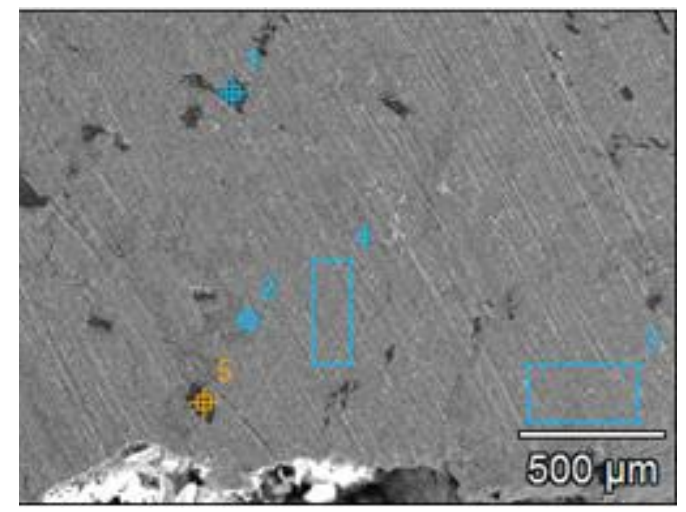

Figura 3. Imagem SEM-EDS do didímo obtido na redução calciotérmica.

Tabela 3.Análise SEM-EDS dos pontos e áreas identificadas na Figura 3.

\begin{tabular}{|c|c|c|c|c|}
\hline \%massa & Al & Ca & Pr & Nd \\
\hline Ponto 1* & $0.2+/-0.2$ & $96.8+/-0.6$ & $1.4+/-0.3$ & $1.6+/-0.3$ \\
\hline Ponto 2 & $3.4+/-0.3$ & $0.1+/-0.1$ & $36+/-2$ & $61.5+/-2$ \\
\hline Área 3 & $0.2+/-0.2$ & $0.8+/-0.1$ & $41+/-2$ & $58+/-2$ \\
\hline Área 4 & $0.2+/-0.2$ & $0.8+/-0.1$ & $42+/-1$ & $57+/-2$ \\
\hline Ponto 5* & 0 & $87+/-0.6$ & $5+/-0.4$ & $8+/-0.5$ \\
\hline
\end{tabular}

* Precipitados escuros da imagem

Outros elementos não foram incluídos na análise SEM-EDS pelo fato do propósito dessa técnica ter sido utilizada para se observar fases precipitadas, representadas pelos pontos 1,5 e 2. Porém, outros elementos foram identificados, tal como silício, magnésio e outros elementos de terras raras. As principais impurezas foram quantificadas por ICP-MS, como apresentado na Tabela 4. 
Tab 4. Análise química do didímio (ICP-MS)

\begin{tabular}{|c|c|}
\hline & \%massa \\
\hline $\mathbf{A l}$ & 0.16 \\
\hline $\mathbf{S i}$ & 0.08 \\
\hline $\mathbf{M g}$ & 0.37 \\
\hline $\mathbf{C a}$ & 0.77 \\
\hline $\mathbf{P r}$ & 49.5 \\
\hline $\mathbf{N d}$ & 49.1 \\
\hline $\mathrm{REE}$ & 0.02 \\
\hline
\end{tabular}

A concentração de carbono e oxigênio foi determinada como de $410 \pm 60$ e $20 \pm 10$ $\mathrm{ppm}$, respectivamente. A alta concentração de carbono provavelmente pode ser atribuída a contaminação de cálcio. $\mathrm{O}$ tratamento a vácuo de $\mathrm{Nd}$ ou didímio contaminado com $\mathrm{Ca}$ e $\mathrm{Mg}$ mostrou que é possível reduzir esses elementos a valores abaixo de $450 \mathrm{ppm}$ de $\mathrm{Ca}$ e 70 ppm de $\mathrm{Mg}$.

Esses resultados podem ser otimizados controlando o nível de vácuo e o tempo de refino, mas demonstram a possibilidade de remoção de $\mathrm{Ca}$ e $\mathrm{Mg}$ por tratamento a vácuo.

A concentração de Al encontrada no metal produzido não é prejudicial aos imãs de $(\mathrm{Nd}, \mathrm{Pr}) \mathrm{FeB}$, considerando que esse elemento é desejado na microestrutura da liga. Já silício deve ser controlado pela pureza das matérias-primas utilizadas.

\subsection{Voltametria Cíclica.}

A solubilidade dos óxido de terras raras em sais de fluoretos fundidos é baixa[8]. Assim, para entender o comportamento voltamétrico do óxido de didímio foi necessário estudar sistemas contendo óxido de didímio ( $\left.\mathrm{LiF}-\mathrm{CaF}_{2}-\mathrm{NdF}_{3}-\mathrm{Nd}_{2} \mathrm{O}_{3}\right)$ e óxido de praseodímio ( $\mathrm{LiF}-\mathrm{CaF}_{2}-\mathrm{PrF}_{3}-\mathrm{Pr}_{11} \mathrm{O}_{6}$ ) separadamente.

A Figura 4 mostra a voltametria cíclica do sistema $\mathrm{LiF}-\mathrm{CaF}_{2}-\mathrm{NdF}_{3}-\mathrm{Nd}_{2} \mathrm{O}_{3}$.

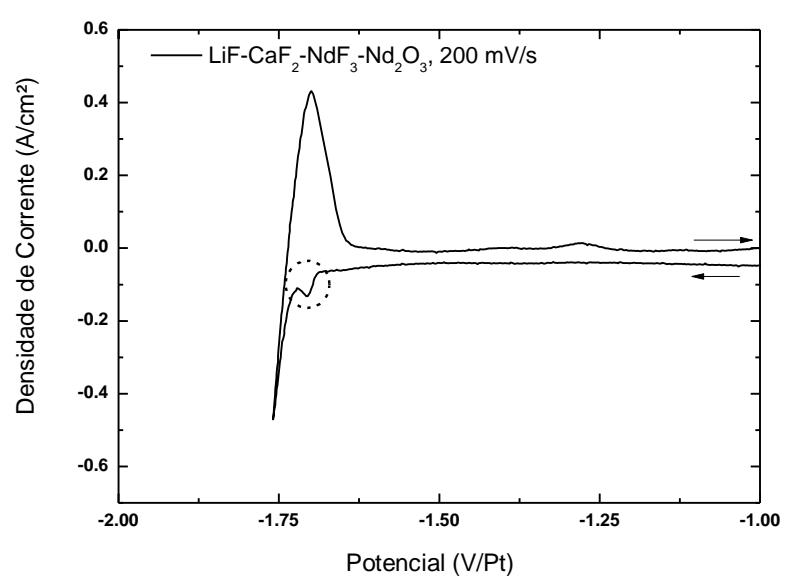


Figura 4. Voltametria Cíclica do sistema LiF-CaF $-\mathrm{NdF}_{3}-\mathrm{Nd}_{2} \mathrm{O}_{3}$ a 200 mV/s.

A Figura 4 mostra a um pico de redução bem definido a $-1,70 \mathrm{~V} / \mathrm{Pt}$ (destacado pelo circulo pontilhado), o qual indica a redução do íon $\mathrm{Nd}$. O próximo pico acentuado a 1,75 V/Pt é um indicativo da redução dos íons $\mathrm{Li}$ e $\mathrm{Ca}$. Na varredura inversa, é possível observar um pico largo o qual indica a oxidação dos íons $\mathrm{Li}$, $\mathrm{Ca}$ e $\mathrm{Nd}$. Contudo, a redução do $\mathrm{Nd}$ se mostra claramente em um único pico, o que sugere que o processo de redução ocorre em uma única etapa.

A Figura 5 mostra a voltametria cíclica do sistema LiF-CaF2-PrF $-\mathrm{Pr}_{11} \mathrm{O}_{6}$.

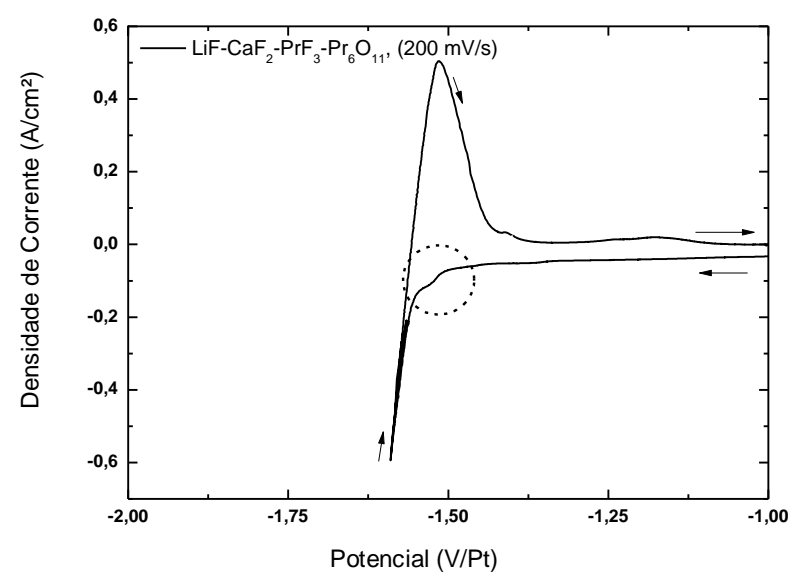

Figura 5. Voltametria Cíclica do sistema LiF-CaF2- $\mathrm{PrF}_{3}-\mathrm{P}_{11} \mathrm{O}_{6}$ a $200 \mathrm{mV} / \mathrm{s}$.

$\mathrm{O}$ voltamograma do sistema $\mathrm{LiF}-\mathrm{CaF}_{2}-\mathrm{PrF}_{3}-\mathrm{Pr}_{11} \mathrm{O}_{6}$ apresenta um limite catódico inferior $(-1,60 \mathrm{~V} / \mathrm{Pt})$ do que o observado no sistema $\mathrm{LiF}-\mathrm{CaF}_{2}-\mathrm{NdF}_{3}-\mathrm{Nd}_{2} \mathrm{O}_{3}$. É possível notar um pico não tão bem definido a $-1,55 \mathrm{~V} / \mathrm{Pt}$ (destacado pelo circulo pontilhado) ao qual corresponde à redução dos íons $\operatorname{Pr}$. O $\operatorname{Pr}_{6} \mathrm{O}_{11}$ é composto pelos íons $\mathrm{Pr}^{4+}$ e $\mathrm{Pr}^{3+}$ e esta pode ser a razão do porquê os picos de redução não serem bem definidos. Provavelmente, a forma do pico está sendo afetada pelas duas reduções simultâneas. A varredura inversa do voltamograma do sistema $\mathrm{LiF}^{-\mathrm{CaF}_{2}-}$ $\mathrm{PrF}_{3}-\mathrm{Pr}_{11} \mathrm{O}_{6}$ é similar ao do sistema com $\mathrm{Nd}$ em sua composição.

A Figura 6 mostra o voltamograma cíclico do sistema LiF-CaF $2-\mathrm{PrF}_{3}-\mathrm{Nd}_{2} \mathrm{O}_{3}-\mathrm{Pr}_{11} \mathrm{O}_{6}$. 


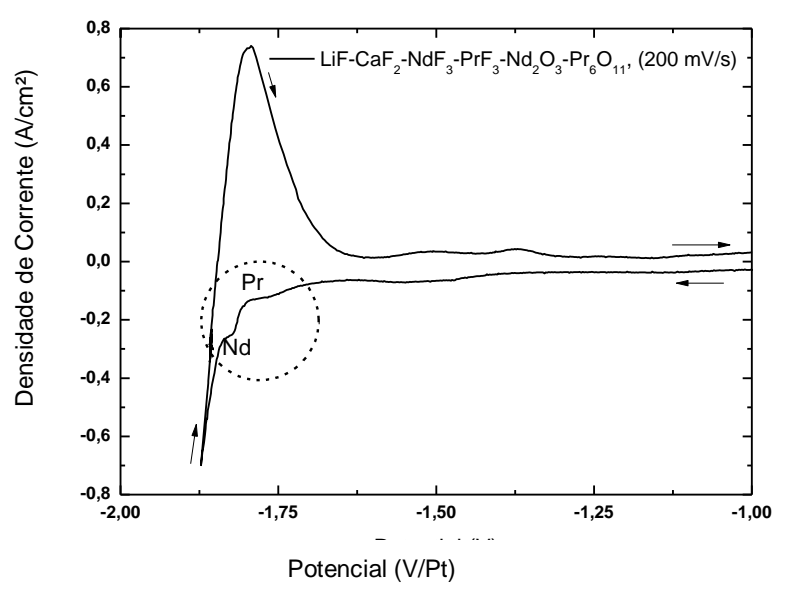

Figura 6. Voltametria Cíclica do sistema LiF-CaF $2-\mathrm{NdF}_{3}-\mathrm{PrF}_{3}-\mathrm{Nd}_{2} \mathrm{O}_{3}-\mathrm{Pr}_{6} \mathrm{O}_{11}$ a 200 $\mathrm{mV} / \mathrm{s}$.

O círculo pontilhado da Figura 6 nostra dois picos de redução. O primeiro indica a redução do $\mathrm{Pr}$, enquanto o segundo indica a redução do $\mathrm{Nd}$. Nota-se que ambos os íons de terras raras reduzem a potenciais muito próximos. A varredura inversa mostrou comportamento similar aos outros voltamogramas.

Os voltamogramas cíclicos mostraram o $\mathrm{Nd}$ reduzindo em uma única etapa, enquanto que o Pr reduz em duas etapas.

\subsection{Redução Eletroquímica}

Foram realizados experimentos o efeito da taxa de alimentação de óxido de didímio na estabilidade do processo. A Figura 7 mostra uma curva típica de corrente e potencial geradas durante eletrólise após a dissolução de $1 \%$ de óxido de didímio no sal fundido. As linhas tracejadas mostram o início (IA) e o final da alimentação (FA) do óxido de didímio. Após um tempo em que o óxido já não era mais alimentado, o experimentou continuou por 20 minutos até a primeira ocorrência de efeito anódico, o qual é representado pela queda abrupta da corrente. A estabilidade do processo só foi novamente alcançada ajustando a corrente para valores menores do que as iniciais, com perda de produtividade. Como se pode observar na Figura 7, este comportamento se repete sucessivamente até a operação ser suspensa por desativação da fonte de corrente contínua. Esse comportamento mostra quão importante é o controle da taxa de alimentação do óxido de didímio no controle apropriado do processo eletroquímico. 


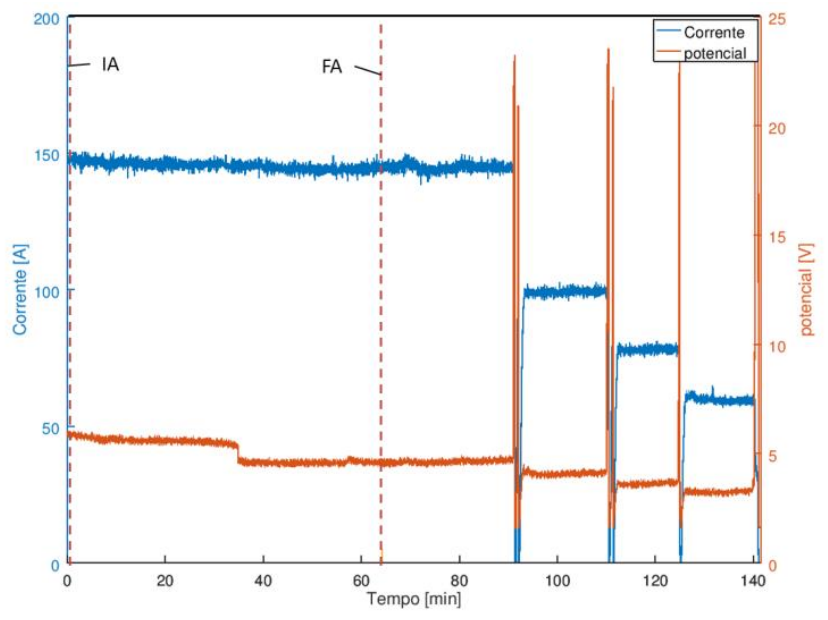

Figura 7. Corrente e Potencial medidos durante redução eletrolítica do óxido de didímio. IA indica o ínicio da alimentação do óxido de didímio e FA indica o final.

A Figura 7 mostra que o processo permanece estável por 90 minutos a $150 \mathrm{~A}$. como a concentração de óxido de didímio aumenta no banho, o potencial entre os eletrodos diminui de 5,2 $\mathrm{V}$ para 4,8 $\mathrm{V}$, indicando um decréscimo na resistividade do banho. Provavelmente, a taxa de alimentação do óxido era maior do que a taxa de redução, o que levou ao aumento da concentração de óxido no eletrólito. Quando a matéria-prima no silo acabou, o processo continuou a operar a condições estáveis por 20 minutos antes da produção do didímio metálico reduzir o óxido a concentrações no banho a valores limites para a corrente ajustada. Nesse momento, a resistividade do banho aumentou e o potencial aumentou para valores superiores a $20 \mathrm{~V}$.

O aumento repentino na resistência é atribuído ao efeito anódico $[9,10]$, ao qual a baixa atividade de óxido leva a redução dos fluoretos de terras raras no sal fundido e a formação de perfluorcarbonos no anodo. Esses gases formam um filme entre o eletrólito e o anodo diminuindo a condutividade elétrica. Além do mais, foram medidos potenciais entre 0,8 e $1 \mathrm{~V}$ entre $\mathrm{o}$ anodo e o eletrodo de referência $(\mathrm{Pt})$ durante a estabilidade do processo e, durante o efeito anódico, esses valores superaram os $20 \mathrm{~V}$ enquanto que o potencial entre o catodo e o eletrodo de referência se manteve entre 2,8 e 2,9 V durante as duas condições de processo.

Os gases formados no efeito anódico foram medidos a jusante do processo de eletrólise. Gases perfluorcarbonos foram absorvidos pelo selo hidráulico formando ácido fluorídrico. $\mathrm{O}$ monitoramento do $\mathrm{pH}$ indicaram a possibilidade de terem sido formados $\mathrm{CF}_{4}$ e $\mathrm{C}_{2} \mathrm{~F}_{6}$ durante a eletrólise. Esse método de medição não é o adequado para quantificar a taxa de produção de perfluorcarbono ou detectar variações dessa produção durante o efeito anódico. A fração volumétrica desses gases foi estimada em quase $10 \%$ dos gases produzido nas células eletroquímicas [11].

$\mathrm{CO}$ e $\mathrm{CO}_{2}$ foram analisados continuamente. A razão $\mathrm{CO} / \mathrm{CO}_{2}$ foi determinada como sendo de aproximadamente 3 , similar a valores reportados por Liu et al. apud Vogel [12], e a produção de $\mathrm{CO}_{2}$ equivalente se manteve entre 0,18 e 0,3 $\mathrm{kg}$ de gás por $\mathrm{kg}$ de TR metálico, exceto pelos perfluorcarbonos não quantificados. 
O metal produzido foi analisado por ICP-MS, atingido pureza superior a $99 \%$, como se pode ver na Tabela 5.

Nota-se uma variação das concentrações de $\mathrm{Nd}$ e $\mathrm{Pr}$ apresentados na Tabela 5. Essas concentrações podem ser controladas pela concentração de fluoretos de neodímio e praseodímio no banho, já que a solubilidade dos óxidos de $\mathrm{Nd}$ e $\operatorname{Pr}$ são afetados pela concentrações dos seus respectivos fluoretos no sal fundido [13].

Tab 5. Composição química do didímio produzido por redução eletroquimica em sais fundidos (ICP-MS).

\begin{tabular}{|c|c|}
\hline & \%massa \\
\hline Al & $0.009-0.015$ \\
\hline $\mathbf{M g}$ & $0.02-0.5$ \\
\hline $\mathbf{C a}$ & $0-0,02$ \\
\hline $\mathbf{P r}$ & $7-44$ \\
\hline $\mathbf{N d}$ & $58-92$ \\
\hline $\mathbf{O}$ & 0.002 \\
\hline ETR & 0.1 \\
\hline
\end{tabular}

A principal impureza encontrada foi magnésio, sua fonte é o $\mathrm{CaF}_{2}$, ao qual foi eliminado quando esse fluoreto deixou de compor a mistura de sais. A eficiência de corrente medida nos diferentes ensaios atingiram valores de até $80 \%$ e o consumo de energia foi estimado entre 3.5 e $4.5 \mathrm{kWh} / \mathrm{kg}$ de didímio.

\section{CONCLUSÃO}

\subsection{Redução Calciotérmica}

Foram obtidas bateladas de didímio metálico por redução calciotérmica. Por essa rota, doi possível obter purezas de até $98,5 \%$ em massa. As principais impurezas encontradas no metal foram alumínio, silício, magnésio e cálcio. A concentração de oxigênio residual encontrada foi menor do que $30 \mathrm{ppm}$. A principal impureza encontrada foi $\mathrm{Ca}$ a $0,8 \%$ em massa, considerando que esse metal é adicionado em excesso para garantir conversão total. A concentração de $\mathrm{Ca}$ e $\mathrm{Mg}$ podem ser reduzidas por refinamento a vácuo.

\subsection{Voltametria Cíclica}

Os ensaios de voltametria cíclica ocorreram em três sistemas de sais fundidos: : LiF$\mathrm{CaF}_{2}-\mathrm{NdF}_{3}-\mathrm{Nd}_{2} \mathrm{O}_{3}$, LiF-CaF2 $-\mathrm{PrF}_{3}-\mathrm{Pr}_{11} \mathrm{O}_{6}$ e $\mathrm{LiF}-\mathrm{CaF}_{2}-\mathrm{NdF}_{3}-\mathrm{PrF}_{3}-\mathrm{Nd}_{2} \mathrm{O}_{3}-\mathrm{Pr}_{6} \mathrm{O}_{11}$. Os voltamogramas cíclicos mostraram que o íon $\mathrm{Nd}$ é reduzido em uma etapa enquanto o íon Pr é reduzido em duas etapas.

\subsection{Redução Eletroquímica}

Didímio metálico com pureza superior a $99 \%$ foi produzido por redução eletroquímica. $\mathrm{O}$ processo eletroquímico é estável quando a concentração de óxido 
no sal fundido é devidamente controlada, pelo controle da taxa de alimentação e pelos parâmetros elétricos da eletrólise (potencial e corrente). Gases anódicos (CO e $\mathrm{CO}_{2}$ ) são continuamente produzidos durante a eletrólise assim como perfluorcarbonos podem também ser gerados. Foi observado que a composição química do eletrólito pode afetar a qualidade do metal e a eficiência do processo.

\section{Agradecimentos}

Os autores agradecem o suporte financeiro da Companhia Brasileira de Metalurgia e Mineração - CBMM e da Empresa Brasileira de Pesquisa e Inovação IndustrialEmbrapii. Os autores também agradecem o apoio técnico às análises químicas pela Agilent e pelo Instituto de Química da Universidade de São Paulo - USP.

\section{REFERÊNCIAS}

1. T. G. Goonan, T.G., 2011, Rare earth elements-End use and recyclability: U.S. Geological Survey Scientific Investigations Report 2011-5094, 15 p. disponível somente em http://pubs.usgs.gov/sir/2011/5094/.

2. Golev, M. Scott, P. D. Erskine, S. H. Ali and G.R. Ballantyne., Rare earths supply chains: Current status, constraints and opportunities, Resources Policy 41 (2014) 52-59.

3. U.S. Geological Survey, 2015, Mineral commodity summaries 2015: U.S. Geological Survey, 196 p., http://dx.doi.org/10.3133/70140094.

4. Usos e aplicações de Terras Raras no Brasil: 2012-2030. Brasília: Centro de Gestão e Estudos Estratégicos, 2013 ISBN 978-85-60755-64-6.

5. Filho, B. F. Riffel, C. A. F. Sousa., Some aspects of the mineralogy of CBMM Nobium deposit and mining and pyrochlore ore processing - Araxá, Mg-Brazil, Proceedings of the International Symposium Niobium 2001, Orlando, Florida, USA. Dec 2-5, 2001, 53-65.

6. S. Shaw, R. Watson, Solubility of Calcium in $\mathrm{CaCl}_{2}-\mathrm{CaO}, 214^{\text {th }}$ ECS Meeting, The Electrochemical Society, 2008

7. S. Wang, F. Zhang, X. Liu, L. Zhang, $\mathrm{CaO}$ solubility and activity coefficient in molten salts $\mathrm{CaCl}_{2}-\mathrm{x}\left(\mathrm{x}=0, \mathrm{NaCl}, \mathrm{KCl}, \mathrm{SrCl}_{2}, \mathrm{BaCl}_{2}\right.$ and $\left.\mathrm{LiCl}\right)$, Thermochimica Acta 470, 2008, 105-107

A. Kaneko, Y. Yamamoto, C. Okada, Electrochemistry of rare earth fluoride molten salts, J. Alloys Compd, 193 (1993) 44-46.

8. D. K. Dysinger, J. E. Murphy. Electrochemical of Neodymium from a Molten Oxide-Fluoride Electrolyte. Bureau of Mines U.S. Department of Interiors. Report Investigantions 1994-9504.

9. J. Thonstad, P. Fellner, G. M. Haarberg, J. Hives, H. Kvande, A. Sterten. Aluminium electrolysis: fundamentals of the Hall-Héroult process. AluminiumVerlag Marketing \& Kommunikation GmbH. Düsseldorf. 2001

10. H. Vogel, B. Friedrich. Controlling the rare earth molten salt electrolysis with reduced PFC emissions. Poster in 11th International Rare Earth Conference in Singapore. 2015

11. H. Vogel, B. Friedrich. Development and Research Trends of the Neodymium Electrolysis - A Literature Review. Proceedings of EMC 2015. 
12.X. Guo, Z.H.I. Sun, J. Sietsma, Y. Yang. A semi-empirical model for the solubility of rare Earth oxides in molten fluorides. Ind. Eng. Chem. Res, 55(16), 2016. 4773-4781. 